\section{Diversity and Transparency in (Volunteered) Geoinformation Practices}

GI_Forum 2018, Issue 2

Page: 97 - 102

Short Paper

Corresponding Author:

tilo.felgenhauer@ph-ooe.at

DOI: 10.1553/giscience2018_02_s97

\author{
Tilo Felgenhauer \\ University of Education Upper Austria, Linz, Austria
}

\begin{abstract}
Geoinformation is represented in increasing numbers of different forms, with heterogeneous social and cultural implications. This paper highlights the merits as well as the ambivalence of the social (especially) and political effects of the widespread expansion and growing importance of (volunteered) geographic information. This general overview of the basic symbolic forms in which geoinfomation appears distinguishes between three areas of spatial semantics: while technological systems address space mainly in numerical terms, the user refers to space by ego-centered deixis, while society and culture provide the established everyday language of toponyms. The ma in argument, based on this categorization, stresses the importance of diversity and transparency in acting and interacting with geoinfomation and geomedia in order to promote empowement, education and reflection.
\end{abstract}

\title{
Keywords:
}

volunteered geographic information, critical GIS, interaction practices, interface design, spatial language

\section{The social embedding of geoinformation}

The landscape of the production and use of geoinformation has changed rapidly in the last decade. Not only has the range of the uses of geoinformation expanded in the everincreasing number of contexts of everyday life which refer to geoinformation, there is also an impressive diversity with regard to the forms in which geoinformation appears. These include, for example, digital cartography based on remote sensing; easy-to-access views of the earth in so called geo-browsers (Abend, 2018); tools which provide navigational guidance or even construct an augmented reality (Graham et al., 2013); volunteered geographic information (VGI) in open source online mapping platforms (Poorthuis \& Zook, 2014); crowd-sourced geodata used for health mapping and epidemiology (Richterich, 2018), and, last but not least, the vast amounts of geoinformation that are an implicit element of geotagged content from social media (Bauder, 2018). Along with the widening of this landscape, there is also growing interest from diverse actors, not originally trained and educated in geography or cartography, who now handle and publish geoinformation in 
manifold forms (Caquard, 2014). This leads to the overall, yet preliminary, conclusion that a shift is occurring from expert towards lay geographies. The traditional authorities in map making and cartography are challenged by the many new forums and channels in which geoinformation appears. At first glance, this field appears to be very heterogeneous, less institutionalized or regulated, often with seemingly flat hierarchies and low barriers for outsiders and the general public to participate.

Critical research in geography and GI science focuses on the relevance and impacts of these phenomena in politics, society and culture, and questions such as the following arise: How does open access to geoinformation change the culture of public participation in politics and planning? How does the constant, automated collection of geoinformation potentially influence practices of governance and political regimes? How does a concept such as 'the general public' change its meaning when (geo)information is constantly being disseminated and (re)structured? How is our geographical knowledge or understanding of space affected by the manifold innovations that are related to digital geoinformation?

\section{Emancipation and democratization of and through geoinformation? Issues, controversies, hopes and obstacles}

In the last decade, there has been in-depth analysis of the impacts of the widespread distribution of geoinformation, especially with regards to so-called 'neogeography' and 'volunteered geographic information'. The discussion has pointed out new horizons of geoinformation and innovative practices, as well as critical issues, limitations and risks associated with these profound changes (Elwood, 2006, 2008; Sui et al., 2013).

Positive developments can be summarized under the terms convergence and integration. For example, Michael Goodchild's concept of 'citizen sensors' (Goodchild, 2007) emphasized the potential which lies in the integration of lay people into processes of the production of GI. Informed and educated lay people, equipped with new digital means, support the collection of geoinformation - potentially all over the world. The formerly exclusive contexts of professional cartography for generating and handling geoinformation have been transformed into bodies of geoinformation that are adaptable, open, and easy to access by the public.

These changing conditions need to be framed and guided. 'Spatial citizenship' is based on the idea that these new possibilities have to be supported by new approaches for schools, students and teachers in order to foster a reflexive and emancipated use of geoinformation. The need for GI education in order for today's citizens to benefit from the new technological possibilities is translated into detailed curriculum concepts (Gryl \& Jekel, 2012). Formerly strict, static and mostly non-transparent structures of cartographic authority and control, institutionalized rules of access and communication, and potential (non-)change are now deeply questioned and becoming transformed. A growing public sense of openness, sharing and participation is becoming common. Moreover, there may even be disciplinary convergence in that social and cultural geographers are becoming interested in the field of GI - a field that formerly was investigated more or less exclusively by GI science. In a word, 
convergence and integration can be observed with regard to formerly separated contexts of production and consumption.

On the other hand, in recent years more detailed analysis has revealed issues of divergence and even exclusion as part of the spread of digital geoinformation. In opposition to the usual discussions of short-sighted scenarios and seemingly one-dimensional social/cultural impacts produced by volunteered geoinformation especially, Elwood (2008) outlined the complexity of the matter. Haklay (2013a; 2013b) expressed his skepticism with regard to the expected effects of democratization that would be brought about by the broad availability and application of VGI. To be more specific, the question of 'participation' has to be looked at closely, because 'volunteering' in fact often refers to automatic geodata-transfer from the user's device to online platforms. According to Harvey (2013), the term volunteered geographic information should be used exclusively to refer to practices which are deliberately chosen and conducted by the so-called 'prosumer' or 'produser' of VGI. In addition to these conceptual considerations, social and cultural asymmetries are also to be found. Referring to the example of Jerusalem, Bittner (2014) draws a picture of vast asymmetries in the practical applications of VGI, in relation to ethnicity and segregated cultural milieus in urban contexts. Stephens (2013) highlights gender inequalities in participating in VGI. In sum, the broad expansion of the phenomenon is in fact highly uneven.

\section{Towards diversity and transparency in VGI practices: critical analysis and potential guidelines}

Instead of weighing the pros and cons of 'neogeography' and VGI with regards on the one hand to their effects on the enlightenment of the public, democratization, emancipation and participation, and scenarios of ubiquitous surveillance and alienation on the other, it could be useful to change the focus. A move from evaluation towards the creation of appropriate heuristics could be helpful. Instead of estimating and measuring effects and impacts of GI on culture, society and politics, it could be helpful - given the empirical heterogeneity of the subject - to develop models of interaction which might provide a useful analytical reductionism. Drawing conclusions from such a heuristic approach, an assessment and critique of VGI might be adequately underpinned (see Budhathoki et al., 2010). Consequently, we suggest shifting the focus in empirical research from context to content, which means distinguishing spatial symbols and languages which contain, represent or imply GI but which, at the same time, stand for very different constructions of space and place.

Interactive online maps, geo-browsers, the geo-tagged content of social media, GPScoordinates, place names, ego-centered spatial graphics, the pins in augmented reality apps, the creative 'carto-semiotics' of way-finding devices - all not only address space differently but, at the same time, imply different practices, different conditions of access and comprehension and, ultimately, different potential social and cultural realms of communication. As a first step, these highly heterogeneous forms of spatial representation could be framed using three basic categories which are more or less always present in the interactive content: (i) the technological system, (ii) the user, and (iii) society/culture as the context in which both are situated. These three categories roughly correspond with (i) 
numerical signs (such as code and coordinates), which stand for a technological and algorithmic processing of geoinformation; (ii) deictic expressions, i.e. expressions whose semantic content depends on the particular situation in which the individual utters them, such as left/right or here/there, and (iii), common place names (toponyms), which represent social and cultural conventions on how to refer to a particular place.

Given these schematic distinctions, it would appear plausible that an educated and reflective use of GI could be promoted by using all three different ways of representing and constructing space in a diverse and transparent manner. In order to build states and processes of convergence between actors, technologies, politics, collectives, individuals and infrastructures, it would be most promising to construct channels and practices of interaction which include a broad diversity of symbolic geographies. Furthermore, their explicit and transparent combination in the design of the interface could support the user's understanding of, and reflection on, the relationship between geodata/geoinformation on the one hand, and its visual and semantic shape on the other (in, e.g., maps, language and graphics). Moreover, in order to offer users and producers a particularly insightful, educative and empowering practice in relation to (V)GI, it could be most fruitful to create interface languages and visual designs which represent and communicate both many of the possibilities to address space and their interrelations. For example, an ideal (V)GI interface design would not only include different symbolic geographies (e.g. geo-coordinates, deictic expressions, toponyms), but would also show the user how these different symbolic forms translate into each other.

\section{Conclusion: On the educational value of complexity}

If diverse and transparent symbolic geographies, or interrelated spatial languages, are a key factor in educating and enabling the users and producers of $(\mathrm{V}) \mathrm{GI}$, the current state of (V)GI practices can be evaluated more systematically and critically, especially in this regard. At this point, the important difference between two seemingly similar modes of interaction becomes clearer: 'easy-to-use' vs. 'self-explanatory' (Felgenhauer, 2017, 879-881).

The first, 'easy-to-use', refers to a low threshold for lay people to access and use GI. We currently observe many innovations in (V)GI in this regard, which are carefully designed in order to appeal to potential consumers who have no particular geographic and cartographic knowledge. In order to achieve simplicity and reduction, many interface designs feature immersive, visually engaging and affective content, reduced to ego-centred visualizations of the user's spatial surrounding. In order to achieve this simplicity and an appealing aesthetics, more complex functions, processes and operations have to be hidden from the user. In many cases, interaction is reduced to the consumption of outputs that are as simple as possible even when user-generated content is contributed. This can be recognized most strikingly in the case of augmented-reality applications which translate and insert GI into (mainly) photographic representations instead of more abstract and conventional cartographies.

In my use of the term, the second mode, 'self-explanatory', differs greatly from the ideal of 'easy-to-use' interfaces. Interpreting the term "self-explanatory" literally we could conclude that it would characterize things which reveal something about themselves - which would 
mean, for example, to provide an explanation about their own functioning. While easy-to-use interface designs aim for reduction and simplification, self-explanatory interaction extends the information communicated towards the user and includes actual explanations as to how the technological system works. In this case, not only would an output be generated and presented, but the user could also receive information on the processes which generated it. For example, the ideal interface would reveal transparent interrelations between GPScoordinates, conventional cartographic representation, and ego-centered graphics. They should all be provided to the user with cross-references and interrelations that are as clear as possible. Furthermore, within this ideal scenario users are encouraged to trace and process these interrelations for themselves. This means not reducing the complexity of geoinformation to a simple graphic pin on a map or an increasingly realistic representation of the user's immediate surroundings. Instead, geoinformation could appear in diverse forms as graphic and naturalistic, as well as abstract, numeric or even algorithmic. At some point, interacting with (V)GI should even imply useful (because educational) complications. For example, to translate common toponyms into deictic terms referring to one's own surroundings, or to imagine geolocation just from reading geo-coordinates, may, at first, be difficult. Yet in the long run such activities would provide rewarding experiences for the user, because he/she achieves a deeper understanding and a more self-determined use of (V)GI. With regard to the general question on how (V)GI can lead to a more emancipated, enlightened, informed and politically engaged public, giving people fruitful challenges and insights into the various processes and forms which together constitute (V)GI could be much more promising than limiting their experience to simple output-consumption.

\section{References}

Abend, P. (2018): From map reading to geobrowsing: methodological reconsiderations for geomedia. In: Felgenhauer, T. \& Gäbler, K. (eds.): Geographies of Digital Culture. London/New York: Routledge, 97-111.

Bauder, M. (2018): Using social media as a big data source for research: the example of ambient geospatial information (AGI) in tourism geography. In: Felgenhauer, T. \& Gäbler, K. (eds.): Geographies of Digital Culture. London/New York: Routledge, 39-51.

Bittner, C. (2014): Reproduktion sozialräumlicher Differenzierungen in OpenStreetMap: das Beispiel Jerusalems. Kartographische Nachrichten, 3/2014, 136-143.

Budhathoki, N.,Nedovic-Budic, Z. \& Bruce, B. (2010): An Interdisciplinary Frame for Understanding Volunteered Geographic Information. Geomatica, 64 (1), 11-26.

Caquard, S. (2014): Cartography II: Collective cartographies in the social media era. (Progress Report). Progress in Human Geography, 38 (1), 141-150.

Elwood, S. (2006): Critical issues in participatory GIS: deconstructions, reconstructions, and new research directions. Transactions in GIS, 10 (5), 693-708.

Elwood, S. (2008): Volunteered geographic information: key questions, concepts and methods to guide emerging research and practice. GeoJournal, 72, 133-135.

Felgenhauer, T. (2017): Self-explanatory or just easy-to-use? A theoretical approach to the emancipatory potential of Volunteered Geographic Information (VGI); GeoJournal, 83 (4), 871-884.

Goodchild, M. (2007): Citizens as sensors: the world of volunteered geography. GeoJournal 69 (4), S. 211-221. 


\section{Felgenhauer}

Graham, M., Zook, M. \& Boulton, A. (2013): Augmented reality in urban places: contested content and the duplicity of code. Transactions of the Institute of British Geographers, 38, 464-479.

Gryl, I. \& Jekel, T. (2012): Re-centring Geoinformation in Secondary Education: Toward a Spatial Citizenship Approach. Cartographica, 47 (1), 2-12.

Haklay, M. (2013a): Citizen Science and Volunteered Geographic Information: Overview and Typology of Participation. In: Sui, D., Elwood, S. \& Goodchild, M. F. (eds.): Crowdsourcing geographic knowledge. Volunteered geographic information (VGI) in theory and practice. Dordrecht, New York. 105122.

Haklay, M. (2013b): Neogeography and the delusion of democratisation. Environment and Planning A, 45, 55-69.

Harvey, F. (2013): To Volunteer or to Contribute Locational Information? Towards Truth in Labeling for Crowdsourced Geographic Information. In: Sui, D., Elwood, S. \& Goodchild, M. F. (eds.): Crowdsourcing geographic knowledge. Volunteered geographic information (VGI) in theory and practice. Dordrecht, New York. 31-42.

Poorthuis, A. \& Zook, M. (2014): Spaces of Volunteered Geographic Information. In: Adams, P. \& Craine, J. (eds): Ashgate Research Companion Media Geography, London: Routledge, 311-328.

Richterich, A. (2018): Digital health mapping: big data utilization and user involvement in public health surveillance. In: Felgenhauer, T. \& Gäbler, K. (eds.): Geographies of Digital Culture. London/New York: Routledge, 144-168.

Stephens, M. (2013): Gender and the GeoWeb: divisions in the production of user-generated cartographic information. GeoJournal, 79, 981-996.

Sui, D., Goodchild, M. \& Elwood, S. (2013): Volunteered Geographic Information, the Exaflood and the Growing Digital Divide. In: Sui, D., Elwood, S. \& Goodchild, M. F. (eds): Crowdsourcing Geographic Knowledge: Volunteered Geographic Information (VGI) in Theory and Practice, Dordrecht, 1-12. 